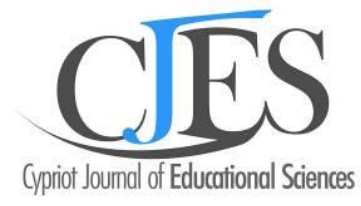

$\underline{\text { www.cjes.eu }}$

\title{
Admission test analysis of pre-service math-science teacher based on its related factors
}

Riskan Qadar a *, Physics Education Study Program, Mulawarman University, Muara Pahu Gn. Kelua, Samarinda 75123, Indonesia, https://orcid.org/0000-0002-4075-4371

Muhamad Arif Mahdiannur ${ }^{\text {b }}$, Science Education Study Program, Surabaya State University, Lidah Wetan, Surabaya 60213, Indonesia, https://orcid.org/0000-0001-9348-139X

Nurul Fitriyah Sulaeman ', Physics Education Study Program, Mulawarman University, Muara Pahu Gn. Kelua, Samarinda 75123, Indonesia, https://orcid.org/0000-0001-5098-0615

\section{Suggested Citation:}

Qadar, R., Mahdiannur, M. A., \& Sulaeman, N. F. (2021). Admission test analysis of pre-service math-science teacher based on its related factors. Cypriot Journal of Educational Science. 16(3), 1154-1174.

https://doi.org/10.18844/cjes.v16i3.5837

Received from January 13, 2020; revised from March 15, 2021; accepted from June 14, 2021.

(C2021 Birlesik Dunya Yenilik Arastirma ve Yayincilik Merkezi. All rights reserved.

\begin{abstract}
The admission test of pre-service teachers uses a knowledge test that has not explored the non-cognitive factors. This study is focused on considering the basic abilities, logical thinking abilities, gender, level of cognitive development and the type of pre-service teacher programme. Moreover, other factors, such as motivation, role of life experiences and verification of logical thinking test result (TOLT), were also contemplated. This study applied TOLT to 281 pre-service math-science teachers from four different programmes. This study also developed a multiple mini interview modification with expert screening methods that have validity, reliability and homogeneity parameters based on Aiken's criteria. Our study provides an alternative to reformulate the selection test that combines paper-based tests with interviews as an admission test for preservice teacher candidates. Our results also illustrate how gender and cognitive development affect the basic abilities based on their programme. Furthermore, admission tests should have balanced cognitive and non-cognitive factors.
\end{abstract}

Keywords: admission test; content-knowledge ability; level of cognitive development; logical thinking (TOLT); multiple-mini interview (MMI); teacher education.

* ADDRESS FOR CORRESPONDENCE: Riskan Qadar, Physics Education Study Program, Mulawarman University, Muara Pahu Gn. Kelua, Samarinda 75123, Indonesia

E-mail address: riskanqadar@fkip.unmul.ac.id/Tel:+6281347849873 


\section{Introduction}

In the era of science and technology innovation, the role of a science teacher became crucial to bring an understanding of the integration of science, technology, mathematics, and engineering (STEM) concepts. The teacher's quality is one of the critical factors in supporting student literacy and the introduction of science for the community to support problem-solving skills in the $21^{\text {st }}$ century (Čipková et al., 2018; Surpless et al., 2014; Woods-McConney et al., 2014). While society expects every student to access satisfactory education, exceptionally qualified teachers (Marco-Bujosa et al., 2019), the teacher education system faces several challenges. Teacher education has been criticized for its week impact on society, the balance between theory and practices courses, ineffective pedagogy, and the low efficacy from teacher candidates (Grossman et al., 2009; König \& Rothland, 2012; Korthagen et al., 2006; Putra \& Kumano, 2018).

On the other hand, most teacher training institutions adopt the college admission test to select the pre-service teacher. The selection test was dominated by the content knowledge divided into two main categories: science and social science. This test was used in most of the Asian countries such as in China, the selection test system called Gaokao (Yang, 2014), South Korea, College Scholastic Ability Test (Suneung) (Kwon et al., 2017), Taiwan (Tuan \& Lu, 2019) and Indonesia (Andreas, 2019) still focused only on content knowledge. The admission test should align with the curriculum and objectives of a program and the practicality and effectiveness of the test (Timarová \& UngoedThomas, 2008). Also, the test needs to acknowledge the cognitive and non-cognitive aspects (Hossler et al., 2019; Makransky et al., 2017). In particular, for pre-service teacher selection tests, the preferred predictors are performance aspect, cognitive aspect, verbal and mathematics aspects (Bieri Buschor \& Schuler Braunschweig, 2018). However, little research has been done before in the selection test that could combine those essential aspects of the specific program in teacher training institutions. Therfore, reformulated the admission test for pre-servise math and science teacher is urgent to select the suitable candidates.

\subsection{Theoretical Framework}

Since it is esensial to nurture addaptive skills on students, preparation of future math and science teacher need to consider not only basic ability but others important skills. To deliver those skills to the students, the teacher needs to possess logical thinking skills. This skill closely related to spatial visualization (Jones et al., 2011) that needed to connect the experience with the process of scientific inquiry (Shepardson \& Britsch, 2001) and mediates the cognitive part with problem-solving skills (Pezzuti et al., 2014). The connection bridge the substantive with procedural concepts. In the context of science-related teaching, logical thinking influences teachers ability to facilitate positive interest toward science (Sezen \& Bülbül, 2011). However, the skills need to earn by a particular process. The level of its development is relatively universal without being significantly influenced by culture, nation-state setting, and geographical aspect (Pogozhina, 2016).

In the addition of the logical thinking part, the measurement of these aspects became the challenge. Studies revealed several ways to measure this skill. The logical thinking test is an instrument that measures formal reasoning and cognitive abilities in a person which consists of five modes, namely, controlling variable, proportional reasoning, combinatorial reasoning, probabilistic reasoning, and correlational reasoning in coordinance with Piaget's developmental theory (Pogozhina, 2016; Tobin \& Capie, 1981; Valanides, 1999). The most common test called the test of logical thinking (TOLT) developed by Tobin \& Capie (1981). Even though initially it was written in English, this test deemed suitable to apply in the developing country (Wilson, 1988). Therefore, we are interested in examining 
the ability of critical thinking of first-year pre-service teachers in the science-mathematics related program and the relation of the result with several factors that support their success in the program. Those factors are the basic ability, logical thinking, gender, cognitive development level, and program choice. In addition to those factors, exploration of their motivation and the role of life experiences were also conducted.

In line with the need to use paper based test, the use of Multiple Mini-Interviews (MMI) could acquire a more comprehensive measurement (Elam et al., 1998; Makransky et al., 2017; Says et al., 2013). MMI is an instrument to assess non-cognitive abilities and proven as reliable tests that widely used (Elam et al., 1998; Makransky et al., 2017; McAndrew \& Ellis, 2012; Says et al., 2013; Yusoff, 2019). To align the MMI with the science-mathematics related pre-service teacher program, clarify attributes of $\mathrm{MMI}$ are need. With $\mathrm{MMI}$, the background such as motivatioan to be a teacher, role of experience and croscheck from TOLT result could be conducted. Therefore, our study provides an alternative way to reformulate the selection test in the pre-service teacher training program that accommodates the logical thinking aspects, especially for science-mathematic related applications. This study also combining paper based test with interview as admission test for pre-service teacher candidate.

\section{Method}

This study was conducted with mixed-method exploratory, which gathered quantitative and qualitative (Hossler et al., 2019), which aimed to evaluate the result of TOLT and examine the formula of the admission test for pre-service science-mathematics related teacher with considering logical thinking ability. The stages in this study were described as follows:

\section{1) Preliminary research}

This preliminary stage was focused on exploring previous research in the admission test for education faculty and the logical thinking test. The purpose of this step is to investigate the instrument's suitability with the respondent's academic background (Sezen \& Bülbül, 2011). After considering the indicators in the instrument and the program characteristics in ScienceMathematics related pre-service teacher program at one of the national university in Indonesia, TOLT that developed by Tobin \& Capie (1981) considered as the most applicable. To clarify the data, MMI was conducted. To align the MMI with the science-mathematics related pre-service teacher program, clarify attributes of $\mathrm{MMI}$ are need. The summary of this attribute could be seen in Table 1.

Moreover, the $\mathrm{MMI}$ attributes were confirmed for its validity and reliability with a screening method involving five experts who are an education faculty member with expertise in pedagogical knowledge, educational psychology, content knowledge, and evaluation in education. The experts were asked to screen the $\mathrm{MMI}$ instrument seven response categories to ensure validity index (V) and reliability with the $\mathrm{R}$ and $\mathrm{H}$ coefficient (Aiken, 1980, 1985). Validity index estimated based on expert assesment through Likert scale. $\mathrm{H}$ coeficient is homogeniety reliability and $\mathrm{R}$ coeficient is reliability coeficient. The coeficient range from 0 to 1 with interpertation that the higher the coeficient is expected. The expert judgement showed that $V_{\mathrm{c}}=0,93, R_{\mathrm{c}}=0,87$, and homogeneity coefficient that indicate the consistency $H_{c}=0,80$ in $95 \%$ confidence level. Comparing those result with statistical table, $V_{\mathrm{c}}>0,77, R_{\mathrm{c}}>0,83$, dan $H_{\mathrm{c}}>0,72$ therefore our instrument proven as feasible instrument. Besides, for approval to use this instrument, ethical approval was achieved from the faculty academic senate. 
Tabel $1 \mathrm{MMI}$ tribute in this study

\begin{tabular}{|c|c|c|}
\hline No. & Attribute & Description \\
\hline 1 & Motivation to be a teacher & $\begin{array}{l}\text { - Desire and confidence to become a teacher } \\
\text { - The deciding factor in choosing the program }\end{array}$ \\
\hline 2 & Role of Life Experiences & $\begin{array}{l}\text { - Understand the central role and attitude of the teaching } \\
\text { profession and how to improve it } \\
\text { - Explore his/her teacher role model } \\
\text { - Explore his/her potential for leadership, collaboration, } \\
\text { and teamwork }\end{array}$ \\
\hline 3 & Exploring TOLT result & $\begin{array}{l}\text { - Especially in problem-solving, critical and creative } \\
\text { thinking, and self-awareness }\end{array}$ \\
\hline
\end{tabular}

\section{2) Group Study}

This study involved first-year students in the academic year 2018/2019 at the department of mathematics and Natural Science in one of National University in Indonesia. Four programs have participated, which are mathematics education, physics education, biology education, and chemistry education. All student participants were 281 students (41 male and 240 female students), ages 17 to 20 years old. In this study, only students of pre-service mathematics and science (biology, chemistry, and physics) education programs considered to be research subjects. However, the entire study group also has STEM-related courses focused on the curriculum of each program.

\section{3) Data Collection}

Several types of data collected, namely national examination data according to students' program; TOLT; and interview data with MMI for some students with high and low category with gender representation. $\mathrm{MMI}$ involves three stations based on the exploration attributes and managed by one interviewer (adapted from McAndrew \& Ellis, 2012).

\section{4) Data Analysis}

The analysis was conducted to observe the trends from the data. Qualitative data were coded and transcripted. Quantitative data were analyzed with Two-Way Multivariate Analysis of Variance (MANOVA) for understanding the effect of gender, student's program choice, the cognitive development level through national exam results, and TOLT score. Both qualitative and quantitative data were triangulated to examine the components of the selection test for preservice science-mathematics related teacher program.

\section{Results}

\subsection{Description of basic abilities of pre-service science-mathematics related teacher program}

The national examination result is one factor that could be seen as our student's basic ability. This test is conducted for $9^{\text {th }}$ graders as a requirement for Senior High School accomplishment. We argue if a pre-service teacher chooses one program (for example, biology), his/her ability in biology 
national exam influences that choice. The data on students' basic ability in science-math related subjects could be seen in Table 2 .

Table 2 Description of Basic Abilities from National Examination Result

\begin{tabular}{lllllllll}
\hline Gender & \multicolumn{2}{c}{ Math } & \multicolumn{2}{c}{ Physics } & \multicolumn{2}{c}{ Biology } & \multicolumn{2}{c}{ Chemistry } \\
& Mean & SD & Mean & SD & Mean & SD & Mean & SD \\
\hline Female & 49.12 & 16.66 & 46.28 & 13.83 & 46.88 & 13.97 & 53.56 & 13.08 \\
Male & 49.08 & 15.83 & 48.94 & 20.52 & 41.55 & 10.98 & 57.00 & 11.52 \\
Total & 48.11 & 16.39 & 46.74 & 14.96 & 46.19 & 13.68 & 53.76 & 12.94 \\
N Subjek & 83 Students & 46 Students & \multicolumn{3}{c}{85 Students } & 67 Students \\
\hline
\end{tabular}

Based on Tabel 2. the distribution of basic ability in four programs is quite low. and the size of concentration is quite large. The national examination in Indonesia has scale $0-100$. therefore total mean around 48.1 is inadequate. The data also showed that students ability is heterogeneous. To understand more detail. Table 3 showed the basic ability of data based on gender. The description based on gender is interesting to observe because in our data the comparison between male and female participants ha quite a large difference (around 1: 6). Consistent with the description in Table 2. the result based on gender also showed that basic ability is not high. Female students are slightly higher than male but gender does not affect the basic ability.

Table 3 Description of Basic Abilities based on gender

\begin{tabular}{ccccc}
\hline Gender & Mean & SD & SE & N \\
\hline Female & 49.14 & 14.70 & 0.95 & 240 \\
Male & 47.81 & 15.51 & 2.42 & 41 \\
Total & 48.95 & 14.80 & 0.88 & 281 \\
\hline
\end{tabular}

\subsection{TOLT results and development of cognitive level}

The results of TOLT based on their program were presented in Table 4 and based on gender in Table 5 . To understand deeply about this result. we analyzed and categorized into three categories which are; (1) concrete thinking level (score 0-1); (2) transitional thinking level (score 2-3); and (3) formal thinking level (score 4-10). The level of cognitive development based on their program could be seen at Tabel 6 dan Tabel 7.

Table 4 Description of TOLT Based on Their Program

\begin{tabular}{lcccccccc}
\hline \multirow{2}{*}{ Gender } & \multicolumn{2}{c}{ Mathematics } & \multicolumn{2}{c}{ Physics } & \multicolumn{2}{c}{ Biology } & \multicolumn{2}{c}{ Chemistry } \\
\cline { 2 - 9 } & Mean & SD & Mean & SD & Mean & SD & Mean & SD \\
Female & 3.89 & 0.19 & 2.97 & 1.80 & 3.01 & 1.34 & 3.40 & 1.85 \\
Male & 4.39 & 2.03 & 3.88 & 1.89 & 4.36 & 2.16 & 3.00 & 0.82 \\
Total & 4.00 & 1.65 & 3.13 & 1.97 & 3.19 & 1.52 & 3.37 & 1.80 \\
N Subject & 83 Students & \multicolumn{4}{c}{ 46 Students } & 85 Students & 67 Students \\
\hline
\end{tabular}

Table 5 Description of TOLT Based on Gender

\begin{tabular}{lcccc}
\hline \multicolumn{1}{c}{ Gender } & Mean & SD & SE & N \\
\hline Female & 3.35 & 1.68 & 0.11 & 240 \\
Male & 4.15 & 1.94 & 0.30 & 41 \\
\hline
\end{tabular}




\begin{tabular}{lllll}
\hline Total & 3.46 & 1.74 & 0.10 & 281 \\
\hline
\end{tabular}

Tabel 6 Description on Cognitive Development Based on Their Program

\begin{tabular}{|c|c|c|c|c|c|c|c|c|c|c|}
\hline \multirow[t]{2}{*}{ Level } & \multicolumn{2}{|c|}{ Mathematics } & \multicolumn{2}{|c|}{ Physics } & \multicolumn{2}{|c|}{ Biology } & \multicolumn{2}{|c|}{ Chemistry } & \multirow[t]{2}{*}{ Total } & \multirow[t]{2}{*}{$\%$} \\
\hline & $\mathbf{M}$ & $\mathbf{F}$ & $\mathbf{M}$ & $\mathbf{F}$ & $\mathbf{M}$ & $\mathbf{F}$ & $\mathbf{M}$ & $\mathbf{F}$ & & \\
\hline Concrete & 1 & 4 & 1 & 11 & 2 & 7 & 0 & 12 & 38 & 13.52 \\
\hline Transitional & 6 & 20 & 3 & 13 & 1 & 47 & 3 & 19 & 112 & 39.86 \\
\hline Formal & 11 & 41 & 4 & 14 & 8 & 20 & 1 & 32 & 131 & 46.62 \\
\hline Total & 18 & 65 & 8 & 38 & 11 & 74 & 4 & 63 & 281 & 100 \\
\hline \multicolumn{11}{|c|}{ M = Male; $F=$ Female } \\
\hline
\end{tabular}

Based on Table 4-6 showed most of the students were at the transitional and formal level. A few students were still in the concrete stage. Interestingly. the results were almost consistent between gender. The deviation of TOLT based on gender is relatively large. It showed cognitive development has not well-developed in high school and this issue needs serious handling. In detail. the modes of reasoning in pre-service teachers based on TOLT result in Table 7.

Table 7 Description of The Reasoning Modes

\begin{tabular}{llcccc}
\hline Gender & Logical thinking mode & Mean & SD & SE & N \\
\hline Female & Reasoning & 0.94 & 0.863 & 0.056 & 240 \\
& Proporsional & 0.10 & 0.306 & 0.020 & \\
& Controlling & 0.35 & 0.616 & 0.040 & \\
& Corelational & 0.46 & 0.500 & 0.032 & \\
& Combinatorial & 1.49 & 0.732 & 0.047 & \\
& Reasoning & 1.15 & 0.792 & 0.124 & 41 \\
& Proporsional & 0.05 & 0.218 & 0.034 & \\
& Controlling & 0.71 & 0.782 & 0.122 & \\
& Corelational & 0.66 & 0.575 & 0.090 & \\
& Combinatorial & 1.59 & 0.741 & 0.116 & \\
& Reasoning & 0.97 & 0.855 & 0.051 & 281 \\
& Proporsional & 0.10 & 0.295 & 0.018 & \\
& Controlling & 0.40 & 0.653 & 0.039 & \\
& Corelational & 0.49 & 0.515 & 0.031 & \\
& Combinatorial & 1.51 & 0.733 & 0.044 & \\
\hline
\end{tabular}

Table 7 provides information that both male and female students have weaknesses in proportional. controlling. and correlation modes. The logical reasoning modes which were quite developed were the reasoning and combinatorial modes. According to the experts. content knowledge in ScienceMathematics related field strongly need to be supported by all modes of reasoning logical thinking as part of human intelligence or soft skills because it is needed in applying scientific process skills and scientific thinking to solve problems both in laboratories and non-laboratories. From the relatively small size of centering data. it indicates the distribution of reasoning modes in student's logical thinking is relatively evenly distributed without any significant difference based on gender 


\subsection{The effect of Gender, Program, and Cognitive Development Level toward Basic Ability and Logical Thinking}

This analysis aimed to provide an overview of gender, program, and cognitive level on the content knowledge and logical thinking skills. As the MANOVA test's prerequisites analysis, the basic ability and TOLT results were converted into Z-score and tested for its normality with the Ryan-Joiner test (Table 8). The results showed that the data were normally distributed and feasible for the MANOVA test (Table 9-12).

Tabel 8 Data Normality

\begin{tabular}{lccccc}
\hline \multicolumn{1}{c}{ Data } & N & SD & RJ & P-Value & Result \\
\hline National Exam & 281 & 1 & 0.999 & $>0.100$ & Normal distributed \\
TOLT Score & 281 & 1 & 0.988 & $>0.100$ & Normal distributed \\
\hline
\end{tabular}

Based on the result in Table 9, P-value result $>\mathbf{0 . 1 0 0}$ which is bigger than $\mathbf{0 . 0 5}$ therefore it is normally distribured. Gender and cognitive level development affected the basic ability based on their program and TOLT result. Interestingly. The program they chose was not a factor that affected all the dependent variables examined in this study. The influence of all independent variables on the dependent variable according to the MANOVA test. The result is presented in Table 10.

Tabel 9 MANOVA (I) Result

\begin{tabular}{|c|c|c|c|c|c|c|c|c|}
\hline \multicolumn{9}{|c|}{ Multivariate Tests } \\
\hline \multicolumn{2}{|c|}{ Effect } & $\begin{array}{c}\text { Valu } \\
e\end{array}$ & $\boldsymbol{F}$ & $\begin{array}{l}\text { Hypothes } \\
\text { is } d f\end{array}$ & Error $d f$ & Sig. & $\begin{array}{l}\text { Noncent. } \\
\text { Parameter }\end{array}$ & $\begin{array}{c}\text { Observed } \\
\text { Power }^{b}\end{array}$ \\
\hline \multirow[t]{5}{*}{ Intercept } & Pillai's Trace & .175 & $28.953^{a}$ & 2.000 & 273.000 & .000 & 57.905 & 1.000 \\
\hline & Wilks' Lambda & .825 & $28.953^{\mathrm{a}}$ & 2.000 & 273.000 & .000 & 57.905 & 1.000 \\
\hline & Hotelling's & .212 & $28.953^{\mathrm{a}}$ & 2.000 & 273.000 & .000 & 57.905 & 1.000 \\
\hline & Trace & & & & & & & \\
\hline & $\begin{array}{l}\text { Roy's Largest } \\
\text { Root }\end{array}$ & .212 & $28.953^{a}$ & 2.000 & 273.000 & .000 & 57.905 & 1.000 \\
\hline \multirow[t]{4}{*}{ Gender } & Pillai's Trace & .036 & $5.092^{\mathrm{a}}$ & 2.000 & 273.000 & .007 & 10.185 & .818 \\
\hline & Wilks' Lambda & .964 & $5.092^{\mathrm{a}}$ & 2.000 & 273.000 & .007 & 10.185 & .818 \\
\hline & $\begin{array}{l}\text { Hotelling's } \\
\text { Trace }\end{array}$ & .037 & $5.092^{\mathrm{a}}$ & 2.000 & 273.000 & .007 & 10.185 & .818 \\
\hline & $\begin{array}{l}\text { Roy's Largest } \\
\text { Root }\end{array}$ & .037 & $5.092^{\mathrm{a}}$ & 2.000 & 273.000 & .007 & 10.185 & .818 \\
\hline \multirow[t]{4}{*}{ Program } & Pillai's Trace & .040 & 1.856 & 6.000 & 548.000 & .086 & 11.135 & .694 \\
\hline & Wilks' Lambda & .960 & $1.866^{\mathrm{a}}$ & 6.000 & 546.000 & .085 & 11.195 & 697 \\
\hline & $\begin{array}{l}\text { Hotelling's } \\
\text { Trace }\end{array}$ & .041 & 1.876 & 6.000 & 544.000 & .083 & 11.255 & .699 \\
\hline & $\begin{array}{l}\text { Roy's Largest } \\
\text { Root }\end{array}$ & .040 & $3.662^{c}$ & 3.000 & 274.000 & .013 & 10.987 & .797 \\
\hline \multirow{6}{*}{$\begin{array}{l}\text { Cognitive } \\
\text { development } \\
\text { level }\end{array}$} & Pillai's Trace & .799 & 91.115 & 4.000 & 548.000 & .000 & 364.459 & 1.000 \\
\hline & Wilks' Lambda & .203 & $166.389^{a}$ & 4.000 & 546.000 & .000 & 665.557 & 1.000 \\
\hline & Hotelling's & 3.91 & 266.169 & 4.000 & 544.000 & .000 & 1064.675 & 1.000 \\
\hline & Trace & 4 & & & & & & \\
\hline & Roy's Largest & 3.91 & $535.916^{c}$ & 2.000 & 274.000 & .000 & 1071.833 & 1.000 \\
\hline & Root & 2 & & & & & & \\
\hline
\end{tabular}




\begin{tabular}{|c|c|c|c|c|c|c|c|c|}
\hline \multicolumn{9}{|c|}{ Multivariate Tests } \\
\hline \multicolumn{2}{|c|}{ Effect } & $\begin{array}{c}\text { Valu } \\
e\end{array}$ & $\boldsymbol{F}$ & $\begin{array}{l}\text { Hypothes } \\
\text { is } d f\end{array}$ & Error $d f$ & Sig. & $\begin{array}{l}\text { Noncent. } \\
\text { Parameter }\end{array}$ & $\begin{array}{l}\text { Observed } \\
\text { Power }^{b}\end{array}$ \\
\hline \multirow[t]{5}{*}{ Intercept } & Pillai's Trace & .175 & $28.953^{a}$ & 2.000 & 273.000 & .000 & 57.905 & 1.000 \\
\hline & Wilks' Lambda & .825 & $28.953^{\mathrm{a}}$ & 2.000 & 273.000 & .000 & 57.905 & 1.000 \\
\hline & Hotelling's & .212 & $28.953^{a}$ & 2.000 & 273.000 & .000 & 57.905 & 1.000 \\
\hline & Trace & & & & & & & \\
\hline & $\begin{array}{l}\text { Roy's Largest } \\
\text { Root }\end{array}$ & .212 & $28.953^{a}$ & 2.000 & 273.000 & .000 & 57.905 & 1.000 \\
\hline \multirow[t]{4}{*}{ Gender } & Pillai's Trace & .036 & $5.092^{\mathrm{a}}$ & 2.000 & 273.000 & .007 & 10.185 & .818 \\
\hline & Wilks' Lambda & .964 & $5.092^{\mathrm{a}}$ & 2.000 & 273.000 & .007 & 10.185 & .818 \\
\hline & $\begin{array}{l}\text { Hotelling's } \\
\text { Trace }\end{array}$ & .037 & $5.092^{\mathrm{a}}$ & 2.000 & 273.000 & .007 & 10.185 & .818 \\
\hline & $\begin{array}{l}\text { Roy's Largest } \\
\text { Root }\end{array}$ & .037 & $5.092^{\mathrm{a}}$ & 2.000 & 273.000 & .007 & 10.185 & .818 \\
\hline \multirow[t]{4}{*}{ Program } & Pillai's Trace & .040 & 1.856 & 6.000 & 548.000 & .086 & 11.135 & .694 \\
\hline & Wilks' Lambda & .960 & $1.866^{\mathrm{a}}$ & 6.000 & 546.000 & .085 & 11.195 & .697 \\
\hline & $\begin{array}{l}\text { Hotelling's } \\
\text { Trace }\end{array}$ & .041 & 1.876 & 6.000 & 544.000 & .083 & 11.255 & .699 \\
\hline & $\begin{array}{l}\text { Roy's Largest } \\
\text { Root }\end{array}$ & .040 & $3.662^{c}$ & 3.000 & 274.000 & .013 & 10.987 & .797 \\
\hline \multirow{4}{*}{$\begin{array}{l}\text { Cognitive } \\
\text { development } \\
\text { level }\end{array}$} & Pillai's Trace & .799 & 91.115 & 4.000 & 548.000 & .000 & 364.459 & 1.000 \\
\hline & Wilks' Lambda & .203 & $166.389^{a}$ & 4.000 & 546.000 & .000 & 665.557 & 1.000 \\
\hline & $\begin{array}{l}\text { Hotelling's } \\
\text { Trace }\end{array}$ & $\begin{array}{r}3.91 \\
4\end{array}$ & 266.169 & 4.000 & 544.000 & .000 & 1064.675 & 1.000 \\
\hline & $\begin{array}{l}\text { Roy's Largest } \\
\text { Root }\end{array}$ & $\begin{array}{r}3.91 \\
2\end{array}$ & $535.916^{c}$ & 2.000 & 274.000 & .000 & 1071.833 & 1.000 \\
\hline \multirow{2}{*}{\multicolumn{9}{|c|}{$\begin{array}{l}\text { a. Exact statistic } \\
\text { b. Computed using alpha }=.05\end{array}$}} \\
\hline & & & & & & & & \\
\hline \multicolumn{9}{|c|}{ c. The statistic is an upper bound on F that yields a lower bound on the significance level. } \\
\hline
\end{tabular}

Tabel 10 MANOVA (II) Result

Tests of Between-Subjects Effects

\begin{tabular}{|c|c|c|c|c|c|c|c|c|}
\hline Source & $\begin{array}{c}\text { Dependent } \\
\text { Variable }\end{array}$ & $\begin{array}{l}\text { Type III } \\
\text { Sum of } \\
\text { Squares }\end{array}$ & $d f$ & $\begin{array}{l}\text { Mean } \\
\text { Square }\end{array}$ & $F$ & Sig. & $\begin{array}{l}\text { Noncent. } \\
\text { Parameter }\end{array}$ & $\begin{array}{l}\text { Observed } \\
\text { Power }^{b}\end{array}$ \\
\hline \multirow[t]{3}{*}{ Corrected Model } & NE_score & $44.811^{a}$ & 6 & 7.468 & 8.701 & .000 & 52.206 & 1.000 \\
\hline & $\overline{\text { TOLT }}$ & $226.586^{c}$ & 6 & 37.764 & 193.72 & .000 & 1162.339 & 1.000 \\
\hline & & & & & 3 & & & \\
\hline \multirow[t]{2}{*}{ Intercept } & NE_score & 3.513 & 1 & 3.513 & 4.093 & .044 & 4.093 & .522 \\
\hline & TOLT & 11.293 & 1 & 11.293 & 57.933 & .000 & 57.933 & 1.000 \\
\hline \multirow[t]{2}{*}{ Gender } & NE_score & .513 & 1 & .513 & .598 & .440 & .598 & .120 \\
\hline & TOLT & 1.605 & 1 & 1.605 & 8.235 & .004 & 8.235 & .816 \\
\hline \multirow[t]{3}{*}{ Program } & NE_score & 8.348 & 3 & 2.783 & 3.242 & .023 & 9.726 & .741 \\
\hline & TOLT & .108 & 3 & .036 & .185 & .906 & .556 & .084 \\
\hline & $\begin{array}{l}\text { Cognitive } \\
\text { Developmen } \\
\text { t Level }\end{array}$ & 33.669 & 2 & 16.835 & 19.613 & .000 & 39.225 & 1.000 \\
\hline
\end{tabular}


Tests of Between-Subjects Effects

\begin{tabular}{|c|c|c|c|c|c|c|c|c|}
\hline Source & $\begin{array}{c}\text { Dependent } \\
\text { Variable }\end{array}$ & $\begin{array}{l}\text { Type III } \\
\text { Sum of } \\
\text { Squares }\end{array}$ & $d f$ & $\begin{array}{l}\text { Mean } \\
\text { Square }\end{array}$ & $F$ & Sig. & $\begin{array}{l}\text { Noncent. } \\
\text { Parameter }\end{array}$ & $\begin{array}{c}\text { Observed } \\
\text { Power }^{b}\end{array}$ \\
\hline & TOLT & 208.848 & 2 & 104.424 & 535.67 & .000 & 1071.345 & 1.000 \\
\hline & & & & & 3 & & & \\
\hline \multirow[t]{3}{*}{ Error } & National & 235.189 & 274 & .858 & & & & \\
\hline & Exam Score & & & & & & & \\
\hline & TOLT & 53.414 & 274 & .195 & & & & \\
\hline \multirow[t]{2}{*}{ Total } & $\begin{array}{l}\text { National } \\
\text { Exam Score }\end{array}$ & 280.000 & 281 & & & & & \\
\hline & $\overline{T O L T}$ & 280.000 & 281 & & & & & \\
\hline \multirow[t]{2}{*}{ Corrected Total } & $\begin{array}{l}\text { National } \\
\text { Exam Score }\end{array}$ & 280.000 & 280 & & & & & \\
\hline & TOLT & 280.000 & 280 & & & & & \\
\hline
\end{tabular}

a. $\mathrm{R}$ Squared $=.160$ (Adjusted R Squared $=.142$ )

b. Computed using alpha $=.05$

c. R Squared $=.809$ (Adjusted R Squared $=.805)$

The result in Table 10. it can be seen that gender was not significantly influenced student's basic ability based on the program but has a significant effect on the TOLT results. The choice of program influenced their basic abilities significantly but was not significantly influenced the TOLT result. The level of cognitive development significantly influenced the basic ability according to their program and their TOLT result. In general. Gender and cognitive development level affected the basic abilities according to their program and TOLT score. However. The program was not a factor that influenced all the dependent variables that we explored. Based on the Levene test results for homogeneity of all dependent variables. The basic ability meets the homogeneity criteria (Sig. $0149>$ 0.05). While the TOLT results were not homogeneous. For confirming this result. The Bonferroni test are performed as seen in Tables 11 and 12.

Table 11 Post Hoc I Result

\begin{tabular}{|c|c|c|c|c|c|c|c|}
\hline \multicolumn{8}{|c|}{ Multiple Comparisons } \\
\hline \multirow{2}{*}{ Dependent Variable } & \multirow{2}{*}{ Dept (I) } & \multirow{2}{*}{ Dept (J) } & \multirow{2}{*}{$\begin{array}{c}\text { Mean } \\
\text { Differenc } \\
e(I-J)\end{array}$} & \multirow{2}{*}{$\begin{array}{l}\text { Std. } \\
\text { Error }\end{array}$} & \multirow[b]{2}{*}{ Sig. } & \multicolumn{2}{|c|}{$\begin{array}{c}\text { 95\% Confidence } \\
\text { Interval }\end{array}$} \\
\hline & & & & & & $\begin{array}{l}\text { Lower } \\
\text { Bound }\end{array}$ & $\begin{array}{l}\text { Upper } \\
\text { Bound }\end{array}$ \\
\hline \multirow[t]{9}{*}{ National Exam Score } & Math & Physics & .1601 & .17030 & 1.00 & -.2924 & .6127 \\
\hline & & & & & 0 & & \\
\hline & & Biology & .1974 & .14297 & $\begin{array}{r}1.00 \\
0\end{array}$ & -.1826 & .5773 \\
\hline & & Chemistry & -.3145 & .15216 & .238 & -.7188 & .0899 \\
\hline & & Physics & -.1601 & .17030 & 1.00 & -.6127 & .2924 \\
\hline & & Math & & & 0 & & \\
\hline & & Biology & .0372 & .16958 & 1.00 & -.4134 & .4879 \\
\hline & & & & & 0 & & \\
\hline & & Chemistry & $-.4746^{*}$ & .17740 & .047 & -.9461 & -.0032 \\
\hline
\end{tabular}


Bonferroni

Multiple Comparisons

\begin{tabular}{|c|c|c|c|c|c|c|c|}
\hline \multirow{8}{*}{ Dependent Variable } & \multirow{2}{*}{ Dept (I) } & \multirow{2}{*}{ Dept (J) } & \multirow{2}{*}{$\begin{array}{c}\text { Mean } \\
\text { Differenc } \\
e(I-J)\end{array}$} & \multirow{2}{*}{$\begin{array}{l}\text { Std. } \\
\text { Error }\end{array}$} & \multirow{2}{*}{ Sig. } & \multicolumn{2}{|c|}{$\begin{array}{c}\text { 95\% Confidence } \\
\text { Interval }\end{array}$} \\
\hline & & & & & & $\begin{array}{l}\text { Lower } \\
\text { Bound }\end{array}$ & $\begin{array}{l}\text { Upper } \\
\text { Bound }\end{array}$ \\
\hline & Biology & Math & -.1974 & .14297 & $\begin{array}{r}1.00 \\
0\end{array}$ & -.5773 & .1826 \\
\hline & & Physics & -.0372 & .16958 & $\begin{array}{r}1.00 \\
0\end{array}$ & -.4879 & .4134 \\
\hline & & Chemistry & $-.5118^{*}$ & .15136 & .005 & -.9141 & -.1096 \\
\hline & & $\begin{array}{l}\text { Chemistry } \\
\text { Math }\end{array}$ & .3145 & .15216 & .238 & -.0899 & .7188 \\
\hline & & Physics & $.4746^{*}$ & .17740 & .047 & .0032 & .9461 \\
\hline & & Biology & $.5118^{*}$ & .15136 & .005 & .1096 & .9141 \\
\hline
\end{tabular}

Based on observed means.

The error term is Mean Square(Error) $=.195$.

*. The mean difference is significant at the .05 level.

Table 12 Post Hoc II Result

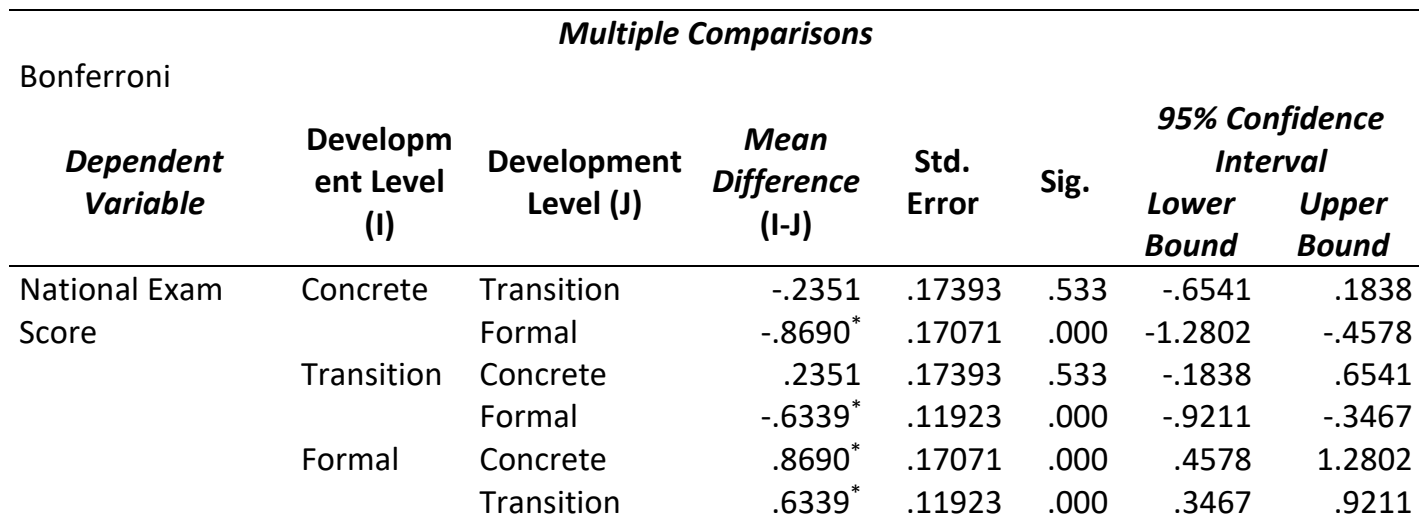

Based on observed means.

The error term is Mean Square(Error) $=.195$.

*. The mean difference is significant at the .05 level.

Table 11 informed significant differences in ability among students from the chemistry, physics and biology program. More specific in Table 12. the result showed significant differences among concrete, transitional, and formal levels.

\subsection{Modified MMI Interview Result}

Based on the qualitative data, the transcription of those interview records from 48 students was developed. Then the transcriptions were coded and matched with the MMI attribute (Table 1). The probes for exploring motivation to became a teacher, the role of life experiences, and crosscheck TOLT results were summarized in Tables 13 and 14. 
Table 13 Summary of Student's Interview: Mathematics Program

\begin{tabular}{cc}
$\begin{array}{c}\text { TOLT score } \\
\text { criteria }\end{array}$ & Gender Summary \\
\hline
\end{tabular}

High

$\mathrm{F}$

Low

M

\section{Motivation to be a teacher;}

- Mostly caused by influence from parents/close relatives;

- Partly caused by recommendation from their teacher;

- Some mentioned hesitation of being a teacher.

Role of Life Experiences:

- Mostly do not have a conception of teacher main task force;

- Mostly have a good impression of their former teacher;

- Mostly chose to work in a team

Crosscheck TOLT Result:

- Mostly have difficulties in propositional mode;

- Mostly assumed TOLT related to mathematics

Motivation to be a teacher;

- Mostly caused by influence from parents/close relatives;

- Mostly have a good impression of math;

- Some students mentioned the hesitation of being a teacher.

Role of Life Experiences:

- Some do not have the conception of teacher main task force;

- Mostly have a good impression of math and math teacher;

- Mostly chose to work individually

Crosscheck TOLT Result:

- Mostly have difficulties in propositional, controlling, reasoning mode;

- Mostly assumed TOLT related to mathematics

Motivation to be a teacher;

- Caused mainly by influence from parents/close relatives and teacher recommendation;

- Mostly do not like math and doubt to be teacher

Role of Life Experiences:

- Mostly do not have a conception of teacher main task force;

- Mostly have a good impression of math and math teacher;

- Mostly chose to work in a team

Crosscheck TOLT result:

- Mostly have difficulties in combinatorial, propositional, correlational, reasoning mode;

- Mostly assumed TOLT related to mathematics 


\begin{tabular}{|c|c|c|}
\hline $\begin{array}{l}\text { TOLT score } \\
\text { criteria }\end{array}$ & Gender & Summary \\
\hline & $F$ & $\begin{array}{l}\text { Motivation to be a teacher; } \\
\text { - Mostly caused by influence from parents/close } \\
\text { - } \text { relatives/teacher; } \\
\text { - Mostly have a good impression of math; } \\
\text { Role of Life Experiences: } \\
\text { - Mostly do not have a conception of teacher main task force; } \\
\text { - Mostly have a good impression to former math teacher; } \\
\text { - } \text { Family/ relatives background have profession as teacher; } \\
\text { - Mostly chose to work in a team } \\
\text { Crosscheck TOLT Result: } \\
\text { - Mostly have difficulties in propositional and correlational } \\
\text { - Mostly assumed TOLT related to mathematics }\end{array}$ \\
\hline
\end{tabular}

Based on the result on Table 13, there were no significant differences between groups and gender that related to motivation to be a teacher (dominated by extrinsic motivation). Moreover, the role of life experiences (commonly do not have a complete image of teacher task force only based on a good impression of their former teacher, especially in high school), and their difficulties in proportional, controlling, correlational, and reasoning. The result for the chemistry, biology, and physics program could be seen in Table 14.

Table 14 Summary of Student's Interview: Chemistry, Biology and Physics Program

\begin{tabular}{|c|c|c|}
\hline $\begin{array}{c}\text { TOLT } \\
\text { score } \\
\text { criteria }\end{array}$ & Gender & Summary \\
\hline High & $M$ & $\begin{array}{l}\text { Motivation to be a teacher; } \\
\text { Chemistry program } \\
\text { - Mostly personal choice and supported by parents/close } \\
\text { - } \quad \text { Molatives; } \\
\text { Biology and Physics program } \\
\text { - Mostly caused by influence from parents/close relatives, they } \\
\text { - } \quad \text { Mave a different interest; } \\
\text { Role of Life Experiences: } \\
\text { Chemistry program } \\
\text { - } \quad \text { Partly understand the description of teacher taskforce; } \\
\text { - Mostly have a good impression to the former teacher; } \\
\text { - Mostly chose to work in a team } \\
\text { Biology and Physics program } \\
\text { - } \quad \text { Partially do not understand the description of teacher } \\
\quad \text { taskforce; }\end{array}$ \\
\hline
\end{tabular}




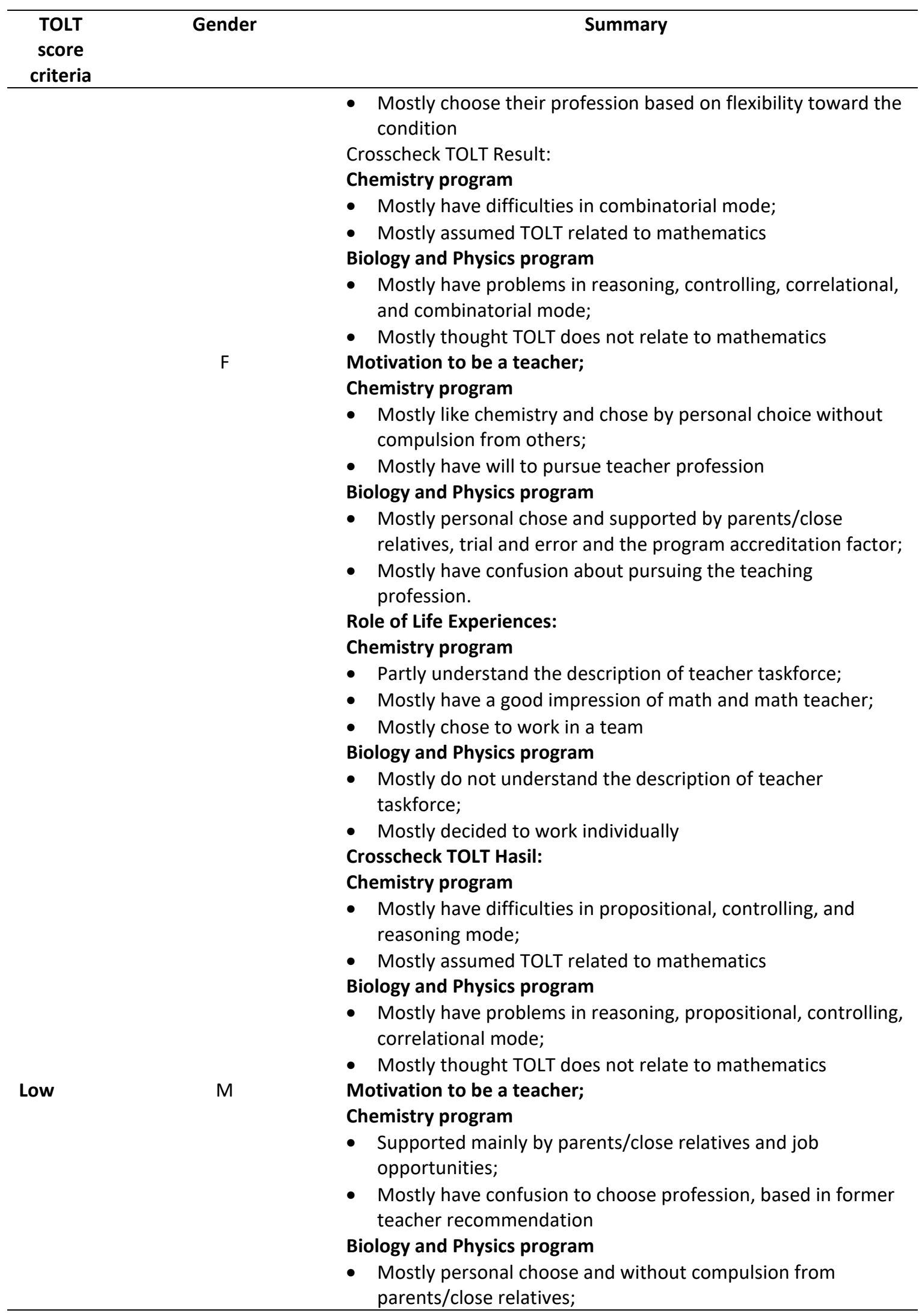




\begin{tabular}{ccc}
\hline TOLT & Gender & Summary \\
score & & \\
criteria & & \\
\hline
\end{tabular}

- Mostly have will to pursue teacher profession

\section{Role of Life Experiences:} Chemistry program

- Mostly do not understand the description of teacher taskforce;

- Mostly have not a good impression to the former teacher;

- Mostly chose to work individually

Biology and Physics program

- Partly understand the description of teacher taskforce;

- Mostly have a good idea to the former teacher;

- Mostly chose to work in a team

Crosscheck TOLT Result:

\section{Chemistry program}

- Mostly have difficulties in combinatorial, propositional, correlational, reasoning mode;

- Mostly assumed TOLT does not relate to mathematics

Biology and Physics program

- Difficulty in all modes especially reasoning;

- Mostly thought TOLT related to mathematics

Motivation to be a teacher;

\section{Chemistry program}

- Mostly supported by parents/close relatives opinion;

- Mostly have not a good impression to chemistry and like another subject;

- Mostly have confusion to pursue teacher profession

Biology and Physics program

- Mostly personal choose and without compulsion from parents/close relatives;

- Mostly have will to pursue teacher profession

Role of Life Experiences:

Chemistry program

- All had not imagined about teacher taskforce;

- Mostly chose to work individually

Biology and Physics program

- All had a description of teacher taskforce;

- Mostly have a good impression to the former teacher;

- Family/close relatives background as teacher;

- Mostly chose to work in a team

\section{Crosscheck TOLT Result:}

\section{Chemistry program}

- Mostly have difficulties in propositional, controlling, correlational, reasoning mode; 


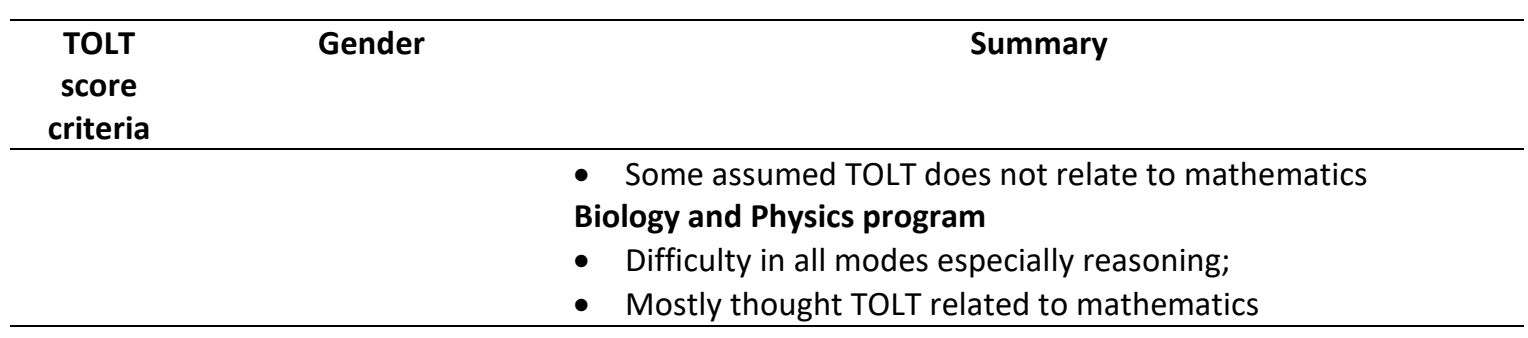

Table 14 showed the contradictory results between the chemistry and biology-physics program. The pattern formed in the chemistry program is linear, which both for male and female students who achieved high TOLT scores tend to have positive results, MMI results, and vice versa. Interestingly, the pattern that formed in Biology and Physics program was oppositely between TOLT results with $\mathrm{MMI}$ results. Both males and females who had a high TOLT result had less positive MMI results.

\section{Discussion}

4.1. Relation Among Basic ability, logical thinking ability, gender, cognitive development level, and their program

One of the essential competencies for teachers that recognized internationally is the content knowledge part. This knowledge can be assumed as the essential ability that coordinance with each student's program. The level of basic ability in our participants tends to be low and spread widely. This result also reinforces that our pre-service teacher's basic ability is low, and the teaching profession is not in high demand by high-achieving students. Gender factors also affect the differences in the basic ability of pre-service teachers. Mastery of content knowledge is inseparable from the characteristics of a professional teacher.

Content knowledge is also influenced by the modes of reasoning in logical thinking. The measurement result of the student's logical thinking using TLT also provides a fascinating insight. On average, students' logical thinking ability is around 3.5 (scale $0-10$ ), which is is categorized as low. Some students were in the concrete and transition level. The consistent result could be observed both in male and female students. These implied two critical points which are (1) learning process in especially at the middle school level, has not been able to create a learning atmosphere that supported the specific level of student's cognitive development; and (2) the existence of students who are at the concrete and transitional level will be an obstruction to internalize the substantive concepts that tend to be abstract and complex. In general, a sufficient level of logical thinking will develop in line with adulthood and supported by collaborative learning that is suitable to inquiry and teacher support (Duncan Seraphin et al., 2017; Shanahan \& Bechtel, 2020; Yu \& Singh, 2018).

The majority of students have reached the formal thinking level based on the further analysis on achieving the level of student cognitive development. A few students are still in the concrete and transition stages. These results indicated that most students could follow the entire lecture process, which used more abstract patterns and higher-order thinking. Moreover, based on the gender factor, there is no effect of learning on differences in students' TOLT scores. Based on Piaget's theory of individual cognitive development, the development of concrete and formal operational level depends on each individual who determines their mental development. Piaget's theory is proven by discovering students who are still in the concrete and transitional operation level. Also, it appears that gender does not significantly affect the cognitive development of university students. 
Although the majority of students were at the formal development level that able to develop reasoning in hypothetical-deductive, some students that still in concrete and transitional development level also need special attention from lectures in pre-service teacher programs. The learning process needs to accommodate all students with their cognitive development levels. Therefore, designing the education system for pre-service teachers is a challenge, especially considering the cognitive abilities and logical thinking level of the new students, which are relatively low and not entirely at the stage of formal operational development. On the other hand, professional science-mathematics-related teachers are demanded to introduce substantive concepts in science-mathematics along with its process that is strongly related to problem-solving based on inquiry and the scientific method. To achieve those demands, pre-service science teachers should have five reasoning modes. (Tobin \& Capie, 1981).

Discussion about gender always interesting to explore further. Our result showed that male and female students have lower weaknesses in proportional, controlling, and correlational mode. The MMI result also showed the same pattern (see Tables 13 and 14). The weaknesses in those modes reinforced the assumption that science-mathematics-related learning in elementary, middle school levels only focused on traditional methods without supported by sufficient inquiry processes and laboratory activities that is the essential central part of the nature of science and mathematics concepts development. Emphasizing the logical mode for pre-service teachers is vital to building teaching pedagogical content knowledge (TPACK) that essential for professional teachers.

This study revealed some impressive results, which are (1) gender and cognitive development level positively affect the basic ability based on the program and TOLT score, but the program is not an influential factor toward all independent variables in this research; (2) there is the significant difference among the programs, especially among chemistry and biology program; and (3) the differences of content ability based on their program is observable on all cognitive development level. Based on those results, gender composition, programs, and cognitive development levels are attribute factors inherited in pre-service teachers. Gender factor is generally associated with individual ability based on gender. This factor also a specific element that urges to have a dominant influence in the education field. In contrast with this assumption, our result showed a consistent effect between males and females.

\subsection{Reformulation Proposal for The selection Test of Science-Mathematics Pre-service Teacher Program}

Annually, the selection test for national universities in Indonesia is accessible through admission possibilities: the national test, regional test, university test, and exclusive cooperation agreement. Pre-service students could apply based on their personal preferences based on their interests, talents, basic ability, and cognitive development level. Specifically, in the ScienceMathematics education program, the characteristic of this program is unique because it will be combining the content knowledge (for example, mathematics knowledge) with pedagogical knowledge. Based on those characteristics, pre-service students that choose this program should have those qualifications to be professional science-mathematics teachers. According to Piaget's theory, cognitive development could be coherent with individual development. This theory also accepted that their cognitive level is at different levels with their biological age in some people. The cognitive development level will affect pre-service teacher performance in their learning process at university. The students will get various tasks that involved their abstract cognition. Our exploration through MMI (Tables 13 and 14) showed that first-year students in the science-mathematics-related program had 
difficulties in several reasoning modes. Students who had a low logical thinking level tend to have problems in all reasoning modes. Each program in the mathematics and natural sciences departments has the same entry requirements and requires all types of reasoning modes. Although the four departments shared similarity, mathematics program has the specific substantive concept that related to fundamental concepts that support science, technology, and engineering. Moreover, physics, chemistry, and biology develop specialties in science that derives from observing natural phenomena from different perspectives. Those characteristics confirmed that TOLT should be part of the selection test as an additional part of the potential scholastic test and academic competency test. In addition to the cognitive part, the selection test also needs to consider the non-cognitive part.

Moreover, although the interview was used, traditional interviews were less focused on the non-cognitive part. We argue that MMI, widely used in the health education sector (Satterfield et al., 2018), could be a powerful instrument in the education program. Our result supports this discussion, which showed the MMI result is coherent with our quantitative data. We could qualitatively describe the different results based on the program (see Table 13 and Table 14). When a teacher teaches mathematics and science at school, he/she need to be actively constructed and develop their mathematical concept (Chamberlin, 2013; Park, 2019). Moreover, he/she need to consider how to build knowledge and self-efficacy that grow with his/her teaching experiences (Rachmatullah \& Ha, 2019; Rozenszajn \& Yarden, 2014). The model of teacher professional development determined by several factors such as (1) teacher characteristics, (2) content knowledge, (3) pedagogical content knowledge of inquiry, (4) experiences, dan (5) adherence (Duncan Seraphin et al., 2017). Scholastic tests and academic tests will predict the content knowledge and a student's potential to develop in general. The four other factors were not accommodated. TOLT and MMI could describe teacher characteristics, pedagogical content knowledge, experiences, and adherence from the pre-service student based on their reasoning modes, motivation to be a teacher, role of life experiences, crosscheck from TOTL result, and problem-solving ability, self-awareness and scientific thinking.

Based on the MMI result, only students in the chemistry program has a linear model between cognitive and non-cognitive factor. Both male and female students with high TOLT scores tend to have intrinsic motivation to be teachers and support families or close relatives. They also have positive experiences toward former teachers, they tend to choose to work in groups and aware of selfdevelopment. Here are some examples of interview transcripts from the students with high TOLT results.

MHS-026: "I like chemistry the choose chemistry education program based on my own will. Being a teacher is an enjoyable profession. [MMI, June 2019]

MHS-030: "... I am sure that the teaching profession is my dream...working on a team is better because we can share our idea and work together." [MMI, June 2019]

On the contrary, the students who had low TOLT results tend to have low intrinsic motivation. From their responses, their motivation came from others, such as their family or friends. Their experiences were less correlated with the teaching profession. They do not prefer to work in a team and confuse about teacher taskforce. Here are some examples of interview transcripts from the students with low TOLT results.

MHS-021: "...I chose chemistry education because of my parent's influence. They said that in this field, the opportunity to work is large." [MMI, June 2019] 
MHS-27: "I knew that teacher only explaining some topics from the textbook... I chose to work alone because it is faster to make the job done. [MMI, June 2019]

Interestingly, the MMI result for biology and physics programs was contrary to the chemistry program trend. Both male and female students that had a high TOLT score showed low intrinsic motivation. Both programs were the program with the best accreditation level based on the Indonesia government accreditation institution. However, the students described that they still confused about their future carrier as a teacher. They also did not have experience related to the teaching profession, and they had low self-awareness. Otherwise, the students with low TOLT results showed a similar conception with chemistry program students that have high TOLT results. In the mathematics program, both male and female students with all TOLT result categories showed more responses, especially in motivation and role of experiences attributes. Minor differences were described in the difficulties of each student to answer the TOLT.

From all the results, it can be concluded that only in the chemistry program where the students met the criteria that fit the teacher professional development model. In biology, physics, and mathematics program, the students tend to confuse non-cognitive factors. Based on the TOLT result, biology and physics program students achieved lower results than students in the chemistry program. This result showed that TOLT and MMI could measure a student's capacity to be successful in teacher preparation programs with a balance between cognitive and non-cognitive factors. Therefore, the integration of TOLT and MMI is essentials (Duncan Seraphin et al., 2017).

\section{Conclusion}

The main contribution of this study is to obtain the illustration of gender, and the cognitive development level affects the basic ability of pre-service teachers based on their program. Our study provides an alternative to reformulate the selection test that combining paper-based tests with interviews as an admission test for pre-service teacher candidates. Our results also illustrate how gender and cognitive development affect the basic ability based on their program. Furthermore, admission tests should have balanced cognitive and non-cognitive factors.

\section{Suggestions}

The implication of this result is a recommendation to integrate modified TOLT and MMI instruments as part of the selection test. In addition to our findings, especially related to gender factors, further research is needed with an equal number of male and female students. Further research about other attributes related to increasing pre-service teacher professionalism also interesting to explore further.

\section{References}

Aiken, L. R. (1980). Content Validity and Reliability of Single Items or Questionnaires. Educational and Psychological Measurement, 40(4), 955-959. https://doi.org/10.1177/001316448004000419

Aiken, L. R. (1985). Three Coefficients for Analyzing the Reliability and Validity of Ratings. Educational and Psychological Measurement, 45(1), 131-142. https://doi.org/10.1177/0013164485451012

Andreas, D. (2019). Alasan Pembentukan LTMPT yang Jadi Penyelenggara SNMPTN dan SBMPTN - Tirto.ID.

Bieri Buschor, C., \& Schuler Braunschweig, P. (2018). Predictive validity of a competence-based admission test - 
mentors' assessment of student teachers' occupational aptitude. Assessment \& Evaluation in Higher Education, 43(4), 640-651. https://doi.org/10.1080/02602938.2017.1390545

Chamberlin, M. T. (2013). Prospective Teachers' Perspectives on Mathematics Teaching and Learning: Lens for Interpreting Experiences in a Standards-Based Mathematics Course. School Science and Mathematics, 113(8), 369-379. https://doi.org/10.1111/ssm.12042

Čipková, E., Karolčík, Š., Sládková, K., \& Ušáková, K. (2018). What is the level of scientific literacy among geography students studying bachelor's studies in natural sciences? International Research in Geographical and Environmental Education, 27(4), 295-310. https://doi.org/10.1080/10382046.2017.1389044

Duncan Seraphin, K., Harrison, G. M., Philippoff, J., Brandon, P. R., Nguyen, T. T. T., Lawton, B. E., \& Vallin, L. M. (2017). Teaching aquatic science as inquiry through professional development: Teacher characteristics and student outcomes. Journal of Research in Science Teaching, 54(9), 1219-1245. https://doi.org/10.1002/tea.21403

Elam, C. L., Burke, M. M., Wiggs, J. S., \& Speck, D. F. (1998). The Medical School Admission Interview: Perspectives on Preparation. NACADA Journal, 18(2), 28-32. https://doi.org/10.12930/0271-9517-18.2.28

Grossman, P., Hammerness, K., \& McDonald, M. (2009). Redefining teaching, re-imagining teacher education. Teachers and Teaching, 15(2), 273-289. https://doi.org/10.1080/13540600902875340

Hossler, D., Chung, E., Kwon, J., Lucido, J., Bowman, N., \& Bastedo, M. (2019). A Study of the Use of Nonacademic Factors in Holistic Undergraduate Admissions Reviews. The Journal of Higher Education, 127. https://doi.org/10.1080/00221546.2019.1574694

Jones, M. G., Gardner, G., Taylor, A. R., Wiebe, E., \& Forrester, J. (2011). Conceptualizing Magnification and Scale: The Roles of Spatial Visualization and Logical Thinking. Research in Science Education, 41(3), 357368. https://doi.org/10.1007/s11165-010-9169-2

König, J., \& Rothland, M. (2012). Motivations for choosing teaching as a career: effects on general pedagogical knowledge during initial teacher education. Asia-Pacific Journal of Teacher Education, 40(3), 289-315. https://doi.org/10.1080/1359866X.2012.700045

Korthagen, F., Loughran, J., \& Russell, T. (2006). Developing fundamental principles for teacher education programs and practices. Teaching and Teacher Education, 22(8), 1020-1041. https://doi.org/10.1016/j.tate.2006.04.022

Kwon, S. K., Lee, M., \& Shin, D. (2017). Educational assessment in the Republic of Korea: lights and shadows of high-stake exam-based education system. Assessment in Education: Principles, Policy \& Practice, 24(1), 60 77. https://doi.org/10.1080/0969594X.2015.1074540

Makransky, G., Havmose, P., Vang, M. L., Andersen, T. E., \& Nielsen, T. (2017). The predictive validity of using admissions testing and multiple mini-interviews in undergraduate university admissions. Higher Education Research \& Development, 36(5), 1003-1016. https://doi.org/10.1080/07294360.2016.1263832

Marco-Bujosa, L. M., McNeill, K. L., \& Friedman, A. A. (2019). Becoming an urban science teacher: How beginning teachers negotiate contradictory school contexts. Journal of Research in Science Teaching. https://doi.org/10.1002/tea.21583

McAndrew, R., \& Ellis, J. (2012). An evaluation of the multiple mini-interview as a selection tool for dental students. British Dental Journal, 212(7), 331-335. https://doi.org/10.1038/sj.bdj.2012.267

Park, J. (2019). Elementary science teacher education in Korea: past, present, and future. Asia-Pacific Science Education, 5(1), 20. https://doi.org/10.1186/s41029-019-0041-z

Pezzuti, L., Artistico, D., Chirumbolo, A., Picone, L., \& Dowd, S. M. (2014). The relevance of logical thinking and cognitive style to everyday problem solving among older adults. Learning and Individual Differences, 36, 218-223. https://doi.org/10.1016/j.lindif.2014.07.011 
Pogozhina, I. (2016). Comparative Study of the Degree of Relationship between Logical Thinking Operational Structures Development Levels in Russian and Chinese Preschoolers. Procedia - Social and Behavioral Sciences, 233, 492-497. https://doi.org/10.1016/j.sbspro.2016.10.201

Putra, P. D. A., \& Kumano, Y. (2018). Energy Learning Progression and STEM conceptualization among pre-service science teachers in Japan and Indonesia. New Educational Review, 53(3), 153-162. https://doi.org/10.15804/tner.2018.53.3.13

Rachmatullah, A., \& Ha, M. (2019). Indonesian and Korean high school student's disparities in science learning orientations: an approach to multi-group structural equation modeling. Asia-Pacific Science Education, 5(1), 19. https://doi.org/10.1186/s41029-019-0048-5

Rozenszajn, R., \& Yarden, A. (2014). Mathematics and biology teachers' tacit views of the knowledge required for teaching: varying relationships between CK and PCK. International Journal of STEM Education, 1(1), 11. https://doi.org/10.1186/s40594-014-0011-7

Satterfield, C. A., Dacso, M. M., \& Patel, P. (2018). Using multiple mini interviews as a pre-screening tool for medical student candidates completing international health electives. Medical Education Online, 23(1), 1483694. https://doi.org/10.1080/10872981.2018.1483694

Says, F. El, Ayuob, N., Fahmy, A. R., Fayez, F. El, Hasanian, M., \& El Deek, B. (2013). Experience of Establishment of Multiple Mini structure Interview as part of student admission policy at Faculty of Medicine, King Abdulaziz University, 2011-2012. Medical teacher, 35(sup1), S74-S77. https://doi.org/10.3109/0142159X.2013.765543

Sezen, N., \& Bülbül, A. (2011). A scale on logical thinking abilities. Procedia - Social and Behavioral Sciences, 15, 2476-2480. https://doi.org/10.1016/j.sbspro.2011.04.131

Shanahan, M., \& Bechtel, R. (2020). "We're taking their brilliant minds": Science teacher expertize, metadiscourse, and the challenges of teacher-scientist collaboration. Science Education, 104(2), 354-387. https://doi.org/10.1002/sce.21550

Shepardson, D. P., \& Britsch, S. J. (2001). The role of children's journals in elementary school science activities. Journal of Research in Science Teaching, 38(1), 43-69. https://doi.org/10.1002/10982736(200101)38:1<43::AID-TEA4>3.0.CO;2-I

Surpless, B., Bushey, M., \& Halx, M. (2014). Developing Scientific Literacy in Introductory Laboratory Courses: A Model for Course Design and Assessment. Journal of Geoscience Education, 62(2), 244-263. https://doi.org/10.5408/13-073.1

Timarová, Š., \& Ungoed-Thomas, H. (2008). Admission Testing for Interpreting Courses. The Interpreter and Translator Trainer, 2(1), 29-46. https://doi.org/10.1080/1750399X.2008.10798765

Tobin, K. G., \& Capie, W. (1981). The Development and Validation of a Group Test of Logical Thinking. Educational and Psychological Measurement, 41(2), 413-423. https://doi.org/10.1177/001316448104100220

Tuan, H.-L., \& Lu, Y.-L. (2019). Science teacher education in Taiwan: past, present, and future. Asia-Pacific Science Education, 5(1), 15. https://doi.org/10.1186/s41029-019-0044-9

Valanides, N. (1999). Formal reasoning performance of higher secondary school students: Theoretical and educational implications. European Journal of Psychology of Education, 14(1), 109-127. https://doi.org/10.1007/BF03173114

Wilson, A. H. (1988). Logical reasoning ability in Papua New Guinea tertiary science-as measured by the test of logical thinking. International Journal of Science Education, 10(5), 589-593. https://doi.org/10.1080/0950069880100512

Woods-McConney, A., Oliver, M. C., McConney, A., Schibeci, R., \& Maor, D. (2014). Science Engagement and 
Literacy: A retrospective analysis for students in Canada and Australia. International Journal of Science Education, 36(10), 1588-1608. https://doi.org/10.1080/09500693.2013.871658

Yang, G. (2014). Are all admission sub-tests created equal? - Evidence from a National Key University in China. China Economic Review, 30, 600-617. https://doi.org/10.1016/j.chieco.2013.12.002

Yu, R., \& Singh, K. (2018). Teacher support, instructional practices, student motivation, and mathematics achievement in high school. The Journal of Educational Research, 111(1), 81-94. https://doi.org/10.1080/00220671.2016.1204260

Yusoff, M. S. B. (2019). Multiple Mini Interview as an admission tool in higher education: Insights from a systematic review. Journal of Taibah University Medical Sciences, 14(3), 203-240. https://doi.org/10.1016/j.jtumed.2019.03.006 